\title{
Clinical Comparison of a Hearing Aid, a Personal Sound Amplification Product, and a Wearable Augmented Reality Device
}

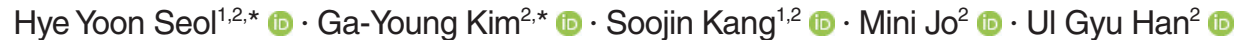 \\ Young Sang $\mathrm{Cho}^{2,3}$ (D) $\cdot$ Sung Hwa Hong ${ }^{2,4}$ (D) $\cdot$ II Joon Moon J $^{2,3}$ (D)

\begin{abstract}
${ }^{1}$ Medical Research Institute, Sungkyunkwan University School of Medicine, Suwon; ${ }^{2}$ Hearing Research Laboratory, Samsung Medical Center, Seoul; ${ }^{3}$ Department of Otorhinolaryngology-Head and Neck Surgery, Samsung Medical Center, Sungkyunkwan University School of Medicine,

Seoul; ${ }^{4}$ Department of Otorhinolaryngology-Head and Neck Surgery, Samsung Changwon Hospital, Sungkyunkwan University School of

Medicine, Changwon, Korea
\end{abstract}

The World Health Organization reported in 2020 that approximately $5 \%$ of the world's population has hearing loss, and stated that one in 10 individuals are expected to be affected by hearing loss due to rapid population aging [1].Although hearing aids (HAs) are the gold standard for hearing loss management, the uptake rate of HAs remains relatively low [2].

The United States Food and Drug Administration defines HAs as medical devices that amplify sounds to compensate for hearing loss. Personal sound amplification products (PSAPs), in contrast, are defined as sound-amplifying devices for those without hearing loss. From traditional HAs to consumer electronic devices, the broad spectrum of "hearables" also includes wearable augmented reality devices (WARDs).WARDs are a combination of smartphone applications and earbuds, providing a personalized listening experience [3]. For instance, the Samsung Galaxy Buds Pro has its own smartphone application called Galaxy Wearable. Users can take advantage of a feature called ambient sound. Similar to how PSAPs are used, individuals can manage the level of sound in their surroundings using WARDs.

Although various types of hearables are emerging in the market, research into the effectiveness of these devices, including WARDs, in the real world has been sparse. The purpose of this

\footnotetext{
- Received February 10, 2021

Revised March 16, 2021

Accepted March 16, 2021

- Corresponding author: Il Joon Moon

Department of Otorhinolaryngology-Head and Neck Surgery, Samsung

Medical Center, Sungkyunkwan University School of Medicine,

81 Irwon-ro, Gangnam-gu, Seoul 06351, Korea

Tel: +82-2-3410-3879, Fax: +82-2-3410-3579

E-mail: moon.iljoon@gmail.com

*These authors contributed equally to this work.
}

study was to explore the effectiveness of a HA, a PSAP, and a WARD with the following four specific aims: (1) to verify the device specifications through electroacoustic testing; (2) to evaluate amplification through simulated real-ear measurements (REMs); (3) to compare unaided and aided performance based on the type of the device; and (4) to evaluate the effectiveness of the WARD in the real world through a field trial.

ReSound LiNX Quattro (a HA; GN Hearing, Ballerup, Denmark), Etymotic Bean (a PSAP; Etymotic Research Inc., Elk Grove Village, IL, USA), and Samsung Galaxy Buds Pro (aWARD; Samsung Electronics Co., Ltd., Suwon, Korea) were used in the study (Supplementary Table 1). Electroacoustic analysis was performed based on ANSI S3.22 (2009) and the previous literature [4-7]. The output sound pressure level (OSPL 90), frequency range, equivalence input noise (EIN), and total harmonic distortion (THD) were tested using the Aurical Hearing Instrument Test box (Natus Medical, Pleasanton, CA, USA) with a HA-2 2cc coupler. Simulated REMs were conducted using a 2cc coupler to evaluate whether the devices provided an appropriate amount of amplification. The target gain calculated by a fitting formula for two virtual audiograms (mild-to-moderate high-frequency and moderate flat hearing loss) and real-ear insertion gain (REIG) calculated through REMs were compared. The PSAP had normal and high modes available and was set to the high mode. The WARD had four levels, of which only level 4 provided amplification; therefore, it was set to level 4 . The amount of amplification was considered adequate if the difference between the target gain and REIG was within $10 \mathrm{~dB}$ at five or more of seven frequencies $(250,500,1,000,2,000,3,000$, 4,000, and 6,000 Hz) [4,6]. Unaided and aided thresholds, word recognition scores in the sound-field (sound-field WRS), and the

Copyright (C) 2021 by Korean Society of Otorhinolaryngology-Head and Neck Surgery.

This is an open-access article distributed under the terms of the Creative Commons Attribution Non-Commercial License (https://creativecommons.org/licenses/by-nc/4.0) which permits unrestricted non-commercial use, distribution, and reproduction in any medium, provided the original work is properly cited. 
Korean version of the Hearing in Noise Test (K-HINT) [8] were also performed.

Eighteen individuals with mild and moderate hearing loss were enrolled in the study. The median age of the participants was 63.0 years (interquartile range [IQR], 59.75-67.25 years). Eleven men $(61.10 \%)$ and seven women $(38.90 \%)$ participated in the study. The degree of hearing loss was determined based on four frequency averages $[(500+1,000+2,000+4,000) / 4 \mathrm{~Hz}]$. The median pure-tone average was $45.00 \mathrm{~dB}$ HL (IQR, 39.38$48.75 \mathrm{~dB}$ HL) in the right ear and $45.63 \mathrm{~dB}$ HL (IQR, 40.00$47.82 \mathrm{~dB}$ ) in the left ear. Demographic data for each participant are shown in Supplementary Table 2. The experiment was carried out on the day that each participant visited our laboratory, and each participant completed all testing wearing the devices. All 18 participants wore the three devices in both ears in serial order, and the order of wearing devices was random. This study was approved by the Institutional Review Board of Samsung Medical Center in Seoul, South Korea (IRB No. 2020-05-052) in accordance with the Declaration of Helsinki. Informed consent was obtained from all participants.

The Friedman test was used to compare the four conditions (unaided, HA, PSAP, and WARD) for the clinical outcomes. The Wilcoxon signed-rank test was utilized for a post-hoc analysis. The Bonferroni correction method with a significance level of 0.0125 (0.05 divided by 4) was applied to determine statistical significance. Internal consistency reliability was assessed using the Cronbach's alpha coefficient. All statistical analyses were completed using IBM SPSS ver. 25 (IBM Corp., Armonk, NY, USA).
The electroacoustic characteristics of all three devices satisfied the tolerances for OSPL $90(<120 \mathrm{~dB}$ SPL), frequency range (250-6,000 Hz), EIN (<28 dB SPL), and THD (<3\%). For both virtual audiograms, all three devices had difference between the target gain and REIG within $10 \mathrm{~dB}$ at five or more of seven frequencies, corresponding to an appropriate amount of amplification. Comparing the unaided and aided thresholds, statistical significance was observed for frequencies at 1,000, 2,000, 4,000, and $6,000 \mathrm{~Hz}$ for HA and PSAP $(\mathrm{P} \leq 0.001)$. Statistical significance was observed at $1,000 \mathrm{~Hz}(P<0.001), 2,000 \mathrm{~Hz}(P=0.001)$, and $6,000 \mathrm{~Hz}(P=0.008)$ for WARD (Table 1$)$. In terms of the sound-field WRS, statistically significant improvements in performance were observed for the HA (median, 92\%), PSAP (median, $88 \%$ ), andWARD (median, $82 \%$ ) compared to the unaided condition (median, 68\%) $(P<0.001)$. No statistical significance was observed for differences in the K-HINT between the unaided and aided conditions for the three devices; however, the PSAP (median, $-0.6 \mathrm{~dB}$ SNR) and WARD (median, $-0.7 \mathrm{~dB}$ SNR) tended to have a better SNR threshold than was observed in the unaided condition (median, $-0.1 \mathrm{~dB}$ SNR) (Table 2). It is difficult to identify what led the PSAP and WARD to have a better SNR threshold, and inconsistent findings have been reported regarding whether there is a difference in SNR thresholds before and after wearing over-the-counter devices. Further studies are necessary to clarify this issue.

The assessment of internal consistency reliability revealed a Cronbach's alpha coefficient of 0.678 , indicating that the questionnaire was consistent and reliable. A frequency analysis of the 5 -point Likert scale revealed that $57.9 \%$ of the participants re-

Table 1. Comparison of unaided and aided thresholds

\begin{tabular}{|c|c|c|c|c|c|c|c|c|}
\hline $\mathrm{Hz}$ & Unaided & $\begin{array}{c}\text { LiNX Quattro } \\
(\mathrm{HA})\end{array}$ & $\begin{array}{l}\text { Bean } \\
\text { (PSAP) }\end{array}$ & $\begin{array}{l}\text { Galaxy Buds Pro } \\
\text { (WARD) }\end{array}$ & $P$-value & $\begin{array}{c}\text { Unaided vs. } \\
\text { HA }\end{array}$ & $\begin{array}{l}\text { Unaided vs. } \\
\text { PSAP }\end{array}$ & $\begin{array}{l}\text { Unaided vs. } \\
\text { WARD }\end{array}$ \\
\hline 250 & $30(20-40)$ & $30(20-36.25)$ & $30(20-40)$ & $25(20-31.25)$ & $0.001^{* *}$ & 0.190 & 0.190 & 0.015 \\
\hline 500 & $35(30-45)$ & $30(30-40)$ & $35(30-43.75)$ & $30(25-40)$ & $0.001^{\star \star}$ & 0.024 & 0.869 & 0.590 \\
\hline 1,000 & $45(40-50)$ & $30(30-35)$ & $35(28.75-45)$ & $35(30-40)$ & $<0.001^{\star \star \star}$ & $<0.001$ & 0.001 & $<0.001$ \\
\hline 2,000 & $50(45-61.25)$ & $37.5(35-41.25)$ & $37.5(30-50)$ & $45(38.75-50)$ & $<0.001^{\star \star *}$ & $<0.001$ & 0.001 & 0.001 \\
\hline 4,000 & $52.5(45-60)$ & $35(30-40)$ & $40(28.75-46.25)$ & $50(40-57.5)$ & $<0.001^{\star * *}$ & $<0.001$ & $<0.001$ & 0.039 \\
\hline 6,000 & $55(50-65)$ & $35(33.75-41.25)$ & $42.5(33.75-55)$ & $55(43.75-60)$ & $<0.001^{\star * *}$ & $<0.001$ & $<0.001$ & 0.008 \\
\hline
\end{tabular}

Values are presented as median (interquartile range).

HA, hearing aid; PSAP, personal sound amplification product; WARD, wearable augmented reality device.

${ }^{\star *} P<0.01,{ }^{* \star \star} P<0.001$. The Bonferroni correction method with a significance level of $0.0125(0.05 / 4)$ was applied to determine statistical significance.

Table 2. Speech measures

\begin{tabular}{|c|c|c|c|c|c|c|c|c|}
\hline Variable & Unaided & LiNX Quattro (HA) & Bean (PSAP) & $\begin{array}{l}\text { Galaxy Buds Pro } \\
\text { (WARD) }\end{array}$ & $P$-value & $\begin{array}{l}\text { Unaided } \\
\text { vs. HA }\end{array}$ & $\begin{array}{l}\text { Unaided } \\
\text { vs. PSAP }\end{array}$ & $\begin{array}{c}\text { Unaided } \\
\text { vs. WARD }\end{array}$ \\
\hline Sound-field WRS (\%) & $68(54-81)$ & $92(84-96)$ & 88 (79-96) & $82(67-90)$ & $<0.001^{\star \star *}$ & $<0.001$ & $<0.001$ & $<0.001$ \\
\hline K-HINT (dB SNR) & $-0.1(-0.95$ to 1.23$)$ & $0.2(-0.95$ to 1.65$)$ & $-0.6(-1.45$ to 0.6$)$ & $-0.7(-1.6$ to 0.1$)$ & 0.090 & - & - & - \\
\hline
\end{tabular}

Values are presented as median (interquartile range).

HA, hearing aid; PSAP, personal sound amplification product; WARD, wearable augmented reality device; WRS, word recognition score; K-HINT, the Korean version of the Hearing in Noise Test; SNR, signal-to-noise ratio.

${ }^{\star \star *} P<0.001$. The Bonferroni correction method with a significance level of $0.0125(0.05 / 4)$ was applied to determine statistical significance. 
ported that the WARD was beneficial in a quiet situation, yet only $26.3 \%$ thought that it was helpful in understanding conversations in a noisy environment.

Our study investigated the effectiveness of three devices (a HA, a PSAP, and a WARD). To the best of our knowledge, this is the first study to investigated and demonstrate a potential benefit of WARD for those with hearing loss. This study demonstrated that along with HAs, PSAPs and WARDs have the potential to be beneficial in a quiet environment for individuals with mild and moderate hearing loss [9]. The results of this study could help these individuals to select appropriate hearing devices, especially when more WARDs are emerging in the market with technological and industrial advances; under such circumstances, it is extremely crucial to closely examine the quality of these devices.

\section{CONFLICT OF INTEREST}

No potential conflict of interest relevant to this article was reported.

\section{ACKNOWLEDGMENTS}

This research was supported by Samsung Electronics Co., Ltd. The authors rented Samsung Galaxy Buds Pro from Samsung Electronics Co., Ltd. for the study.

\section{ORCID}

Hye Yoon Seol

Ga-Young Kim

Soojin Kang

Mini Jo

Ul Gyu Han

Young Sang Cho

Sung Hwa Hong

Il Joon Moon https://orcid.org/0000-0002-7040-1884

https://orcid.org/0000-0002-8945-4927

https://orcid.org/0000-0003-3070-8125

https://orcid.org/0000-0002-8197-811X

https://orcid.org/0000-0001-7743-6214

https://orcid.org/0000-0002-4040-7206

https://orcid.org/0000-0001-6906-8925

https://orcid.org/0000-0002-3613-0734

\section{AUTHOR CONTRIBUTIONS}

Conceptualization: HYS, GYK, SK, MJ, YSC, SHH, IJM. Data curation: HYS, GYK, MJ, UGH. Formal analysis: HYS, GYK, SK, MJ, YSC. Methodology: HYS, GYK, SK, MJ, UGH. Project administration: HYS, GYK, SHH, IJM.Writing-original draft: HYS, GYK, SK. Writing-review \& editing: HYS, GYK, SK, MJ, YSC, SHH, IJM.

\section{SUPPLEMENTARY MATERIALS}

Supplementary materials can be found via https://doi.org/10. 21053/ceo.2021.00297.

\section{REFERENCES}

1. World Health Organization. Deafness and hearing loss [Internet]. Geneva:World Health Organization; 2021 [cited 2021 Jan 7]. Available from: https://www.who.int/news-room/fact-sheets/detail/deafness-and-hearing-loss.

2. Moon IJ, Baek SY, Cho YS. Hearing aid use and associated factors in South Korea. Medicine (Baltimore). 2015 Oct;94(42):e1580.

3. Taylor B. Hearables: the morphing of hearing aids and consumer electronic devices. Audiol Today. 2015;27(6):22-31.

4. Callaway SL, Punch JL. An electroacoustic analysis of over-the-counter hearing aids. Am J Audiol. 2008 Jun;17(1):14-24.

5. Manchaiah V, Taylor B, Dockens AL, Tran NR, Lane K, Castle M, et al.Applications of direct-to-consumer hearing devices for adults with hearing loss: a review. Clin Interv Aging. 2017 May;12:859-71.

6. Reed NS, Betz J, Lin FR, Mamo SK. Pilot electroacoustic analyses of a sample of direct-to-consumer amplification products. Otol Neurotol. 2017 Jul;38(6):804-8.

7. Smith C,Wilber LA, Cavitt K. PSAPs vs hearing aids: an electroacoustic analysis of performance and fitting capabilities. Hear Rev. 2016; 23(7):18.

8. Moon SK, Hee Kim S, Ah Mun H, Jung HK, Lee JH, Choung YH, et al. The Korean hearing in noise test. Int J Audiol. 2008 Jun;47(6): 375-6.

9. Choi JE, Kim J, Yoon SH, Hong SH, Moon IJ. A personal sound amplification product compared to a basic hearing aid for speech intelligibility in adults with mild-to-moderate sensorineural hearing loss. J Audiol Otol. 2020 Apr;24(2):91-8. 